\title{
An agile multi-body additively manufactured soft actuator for soft manipulators
}

Jorge Morales ${ }^{\mathrm{a}}$, Francisco Ramírez $\mathrm{Cruz}^{\mathrm{b}}$, Francisco Eugenio López Guerrero ${ }^{\mathrm{b}}$

${ }^{a}$ Chair of Production Automation and Assembly, University of Siegen, Paul-Bonatz-Str. 9-11, 57076 Siegen, Germany

bUniversidad Autónoma de Nuevo León, Facultad de Ingeniería Mecánica y Eléctrica, Departamento de Mecatrónica

\section{RESUMEN}

Con la introducción de robots colaborativos en entornos de producción, el daño a los trabajadores por el uso de robots tradicionales con enlaces rígidos es inherente. Se ha propuesto una nueva generación de robots hechos de materiales blandos flexibles que reduce el peligro de colisión mediante acciones de autodeformación como una solución prometedora para los entornos de colaboración humano-robot. Recientemente, con el desarrollo de la fabricación aditiva de materiales blandos elásticos, surgen nuevas oportunidades de diseño para estos llamados robots blandos. Sin embargo, aún no se logra la robustez que se requiere para los entornos de producción. Este documento presenta un enfoque de diseño de un actuador neumático blando de tres ejes fabricado de forma totalmente aditiva. Para su uso en sistemas de manipuladores robóticos blandos flexibles, se presentan pautas de diseño, un proceso de impresión $3 D$ directo con materiales elásticos y un sistema de control de regulación de presión semiautomatizado PLC de bajo nivel. Para validar el diseño propuesto, el actuador se fabrica y prueba para la fuerza de contacto máxima, la reacción de movimiento de flexión y su respuesta de señal.

\section{PALABRAS CLAVE}

Materiales blandos, fabricación aditiva, robots blandos, sistema de control.

\begin{abstract}
With the introduction of collaborative robots in production environments, the harm to workers by using traditional robots with rigid links is inherent. A new generation of robots made from flexible soft materials that decreases collision danger by self-deforming actions has been proposed as a promising solution for the human-robot collaboration environments. Recently, by the development of additive manufacture of elastic soft materials, new design opportunities arise for these so-called soft robots. However, robustness that is required for production environments is still not achieved. This paper presents a design approach of a fully additively manufactured three-axis soft pneumatic actuator. For its use in flexible soft robotic manipulator systems, design guidelines, a direct $3 \mathrm{D}$ printing process with elastic materials and a low-level PLC semi-automated pressure
\end{abstract}


regulation control system are presented. To validate the proposed design, the actuator is manufactured and tested for maximum contact force, bending motion reaction and its signal response.

\section{KEYWORDS}

Soft materials, additive manufacture, soft robots, control system.

\section{INTRODUCCIÓN}

In human-robot collaboration environments, where the proximity between human and robot potentially rises a harmful and non-safe working environment. In the case of robot-assisted assembly processes the idea of having a robot operating beside a worker difficult. With the introduction of cobots in the last years, this human-robot collaboration is still not well accepted for workers that claim not feeling safe working together with a robot in the vicinity. The use of soft material robots that can operate in the vicinity without being a threat for the user provide a promising perspective of future shop floor collaboration. Since ${ }^{1}$ pneumatic-driven flexible actuators have been started to be described and their application in certain robotic mechanism.

With the increased and fast emerging development of additive manufacturing technologies, it has been proved that, design and fabrication of complex geometrical structures is possible nowadays. ${ }^{2}$ A more universal way of application is achieved with soft robots because these soft materials, often elastomers, allow them to flexibly adapt to their working environment in terms of conventionally inaccessible work environments (e.g. under sea). ${ }^{3}$ Even more, soft robotic grippers can cling to various shapes and, thus, handle a broader range of applications, as in ${ }^{4}$ were a soft manipulator has been introduced as surgical manipulator. Furthermore, inherently soft, and flexible materials, such as silicon-based polymers, are now available for additive manufacturing.

In industrial and production environments, rigid robots are very well stablished due the robustness and strength capabilities that even small cobots models, can handle several kilograms of payload. In practical robotic applications, the use of end attached tools play a significant role in the mass and speed along a desired motion direction. ${ }^{5}$ Payload is usually a requirement for production processes that cannot be neglected or replaced with the use of soft rubber-like materials. Adding mass to these so called, soft actuators raises the gravitational load, which for in a multi-body soft manipulator configuration, results in larger joint tor-ques and have a big influence in the required energy in order to maintain its deformed position. For pneumatically operated actuators and as investigated in, ${ }^{6,7,8,9,10}$ generated blocked force is still very low for a pneumatically actuated net-work, usually for a single PneuNet, values are in the $3 \mathrm{~N}$ surroundings. For AM soft materials, forces are usually lower than what casted materials can offer. Recent studies as in $^{11}$ analyzes lifting and twisting forces for handling different objects.

However, the lack of material testing standards for AM soft polymers and material models rises the challenges of how-to bring AM soft robots to a performance level to functionally compete with classical rigid robots. Recent works as in, ${ }^{12}$ offer a perspective on mechanical properties and characteristics for different types of elastomers used in soft robotics applications. Nevertheless, durability of available materials represents yet an unanswered question. 


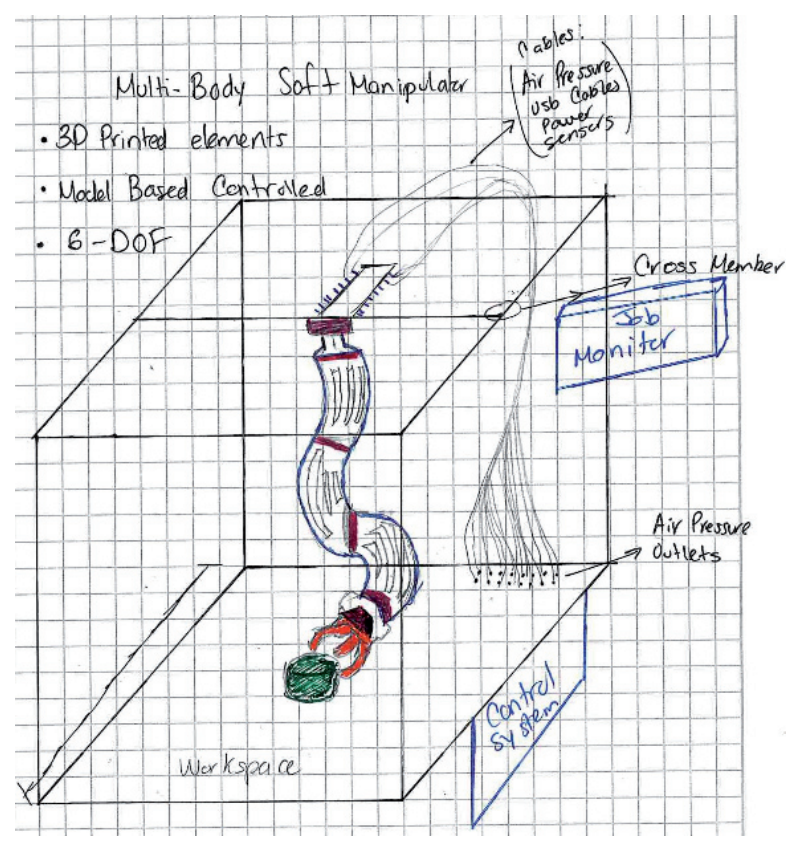

Fig. 1. Conceptual design of a fully additively manufactured soft robotic arm manipulator.

In this work, a three-axis additively manufactured soft silicon-based body segment for a soft manipulator is presented. In order to overcome classic fabrication challenges as presented in, ${ }^{9}$ a uni-body fully additively manufactured segment of a multi-body smart manipulator is investigated. Besides the design challenges inherent to AM, the maximum displacement that the element can achieve, as the maximum force and momentum are measured and analyzed in this work. The aim of this paper is to design and characterize a single fully $3 \mathrm{D}$ printed soft silicon actuator.

Starting from a conceptual sketch for a multi-body flexible manipulator as seen in figure 1, this work presents a new design approach of a unibody element that can be easily manufactured and assembled to form a more complex soft robot arm manipulator. Together with an automated PID low level control and a semi-automated PLC system, the actuator is tested for different pressure set points and the resultant force for each case is also measured.

Based on previous works, ${ }^{13,14}$ we have discussed the advantages and disadvantages of the additive manufacture of soft actuators using silicon-based materials, that together with a new design perspective, targeted for a fully AM fabrication we investigate how fabrication challenges and controllability issues can be handled for the introduction of such actuators as reliable products in production processes. Due to the problems associated with fabrication and assembly of soft material actuators and robots, we focus on the development of a single fully $3 \mathrm{D}$ printed actuator as one element of a manipulator body. Drawing from previous experiences and expertise in additive manufacture of soft materials as in, ${ }^{13,14}$ and in comparison with typical manufactured actuators where shapes and features, due to the complexity of casting, is limited, as presented in ${ }^{8}$ see (figure 2a), we present an innovative three axis design for a unibody soft actuator (see figure $2 b$ ). 

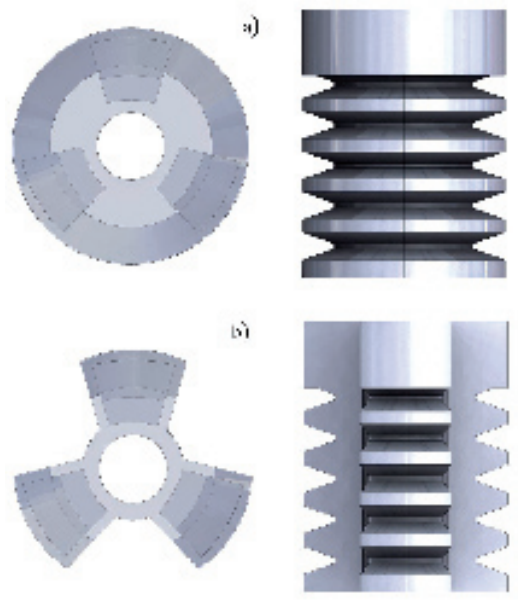

Fig. 2. a) Typical design [7] in conventionally molded fabrication setup (upper parts) and b) chances for AM fabricated actuators (lower parts).

In this paper, we intend to demonstrate the possibility to fully $3 \mathrm{D}$ print a soft actuator, that can be later used to build a fully $3 \mathrm{D}$ printed soft robotic manipulator. An easy-to-build approach and a reliable air pressure supply signal is the key to reduce the fabrication, ${ }^{15}$ and control, ${ }^{16,17}$ complexity that until now, soft robots present. $^{18}$

Traditionally soft robots manufactured by casting, which is usually a long process that, due to fabrication of molds, and the process itself of pouring and curing tend to be time consuming. Molds are already 3D printed, ${ }^{19,20}$ in order to achieve the com-plex geometry forms, that are required for such soft robots. However, the stepwise nature of this classical fabrication method, ${ }^{21,8}$ the material waste and post processing effort that compared in an almost not need of post processing or material waste, as it 3D printing, makes it a unique product manufacture solution at low volumes in a cost-effective way. ${ }^{22}$ Therefore, additive manufacture has the potential to scale better.

Furthermore, 3D printing allows integrating geometrical features in parts to improve functionality. In contrast to classical methods, the main disadvantage of AM yet is the lack of mechanical performance, as known from the conventional silicone-based polymer compounds. As described in, ${ }^{23}$ the type of material has a direct influence on the shape, dimensions, durability, applications and costs of printed parts. Despite problems with durable integrity, the easy manufacture of soft material components for the application in production engineering is advancing. Our study, therefore, evaluates the reliability of the materials used, its motion response and its operational force in a robotic force sensing test as in, ${ }^{24}$ using automated PID regulated air pressure signals.

\section{OBJECTIVES}

This work is focused on if and how a direct AM process can be applied for the fabrication of soft material bio inspired manipulators. We present an easy-to-build uni-body design approach for a modular, multi-body manipulator. In this context, AM enables substitution of several components by integration of their respective functionality. The differential design and construction as well as elaborate 
assembly steps of conventional manufacturing processes is hence avoided. We investigate how a design for AM affects the challenges related to this fabrication process and if such actuators can perform in production process environments. A prototype of a module is built and tested for maximum forces generated and the reaction of automated control signals. A two-element manipulator prototype is investigated for its motion response.

\section{MATERIALS AND METHODS}

The material "agilus30" white from the manufacturer Stratasys was used in this work for fabrication of the soft robotic unit. The mechanical material parameters, as given by Stratasys in, ${ }^{20}$ and are summarized in table I.

Table I. OEM mechanical parameters.

\begin{tabular}{|c|c|c|c|}
\hline Parameter & Unit & white & black \\
\hline Tensile strength & {$[\mathrm{MPa}]$} & $2.1-2.6$ & $2.4-3.1$ \\
\hline Failure elongation & {$[\%]$} & $180-230$ & $220-270$ \\
\hline Density & {$[\mathrm{g} / \mathrm{cm} 3]$} & $1.14-1.15$ & $1.14-1.15$ \\
\hline Shore A hardness & - & $30-40$ & $30-35$ \\
\hline
\end{tabular}

\section{Design for additive manufacture}

Machine developers have been introducing in recent years soft, elastic materials. Such materials arise the challenges even more on how to design functional and reliable components. For designers, who lack design guidelines, standards and manufacture norms are already challenges, ${ }^{25}$ these new materials came with a set of valuable mechanical properties, as is high elasticity and deformation levels. As has been seen in, ${ }^{26,13}$ there are some design requirements that for this work are considering:

- Full removal of internal support materials

- Unibody design

- Easy and fast fabrication

- Lightweight and minimum material usage

- Easy to assembly

- Expected maximum force $\mathrm{F}>=10 \mathrm{~N}$

Figure 3 displays the final design of the actuator. Based on our own previous experience,${ }^{11}$ for design and additively manufacture soft actuator form the PneuNet type a three-chambers unibody design is proposed. Each of the internal chambers as is shown in figure $3 \mathrm{~d}$ is interconnected trough a main channel where, when air fulfill the volume, these tend to expand the chamber with a uniaxial total directional bending motion.

\section{Fabrication process}

Due to the advantages of direct 3D printing technologies, as is the development of new materials, a single two-steps fabrication process was required. Fabrication process presented on this work was made using a Stratasys PolyJet Connex 

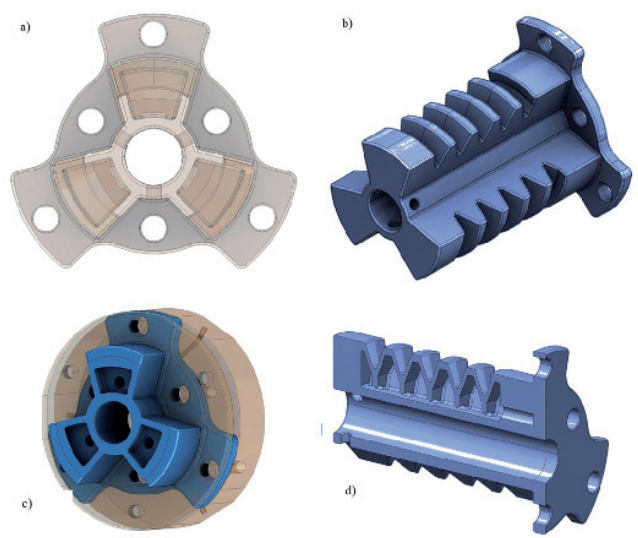

Fig. 3. a) front view of the final actuator design, b) isometric view, c) cut-view of the internal air inlets as is finally assembled, d) cut-view internal chamber and channel.

3 series. Removal of support material is conducted in our laboratory using a potassium hydroxide solution as a solver. Fully removal of support material is still an issue for the deepest internal pneumatic chambers, however, machine manufacturers now offering chemical solutions to react and remove such supports improves significantly the cleaning process. In figure 4, the resulted component is shown.

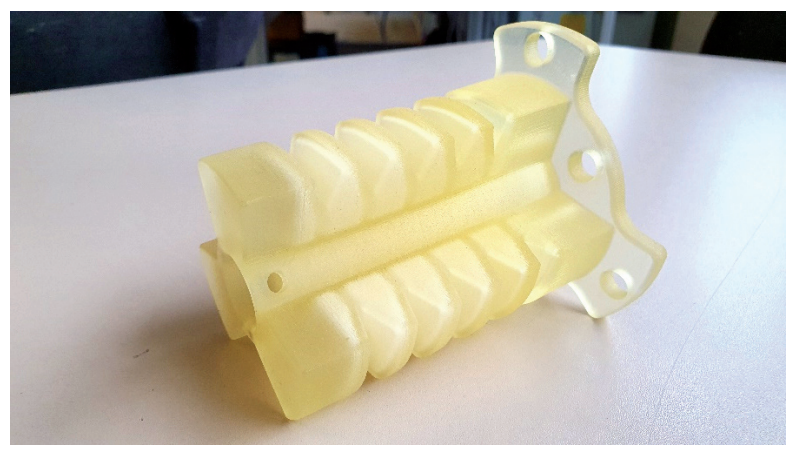

Fig. 4. Final product overview. Support materials are already being removed for a total mass $\mathrm{m}=190 \mathrm{gr}$ and a total length $\mathrm{l}=108 \mathrm{~mm}$.

\section{Bending forces}

The three-axis AM actuator's design essentially resembles a tube with three PneuNets, longitudinally attached under an angle 120 degree towards each other. In order to predict the component's elastic behavior, the structural stiffness needs to be considered. Figure 5 shows the schematic cut view with relevant dimensions of the soft robotic unit.

Mechanically, the component's PneuNets are integrated in a way, so that they do not significantly contribute to stiffness, which, overall, is determined by the centric tube feature. In order to derive the force, which is generated by the actuator, elastic bending stiffness of a tube needs to be considered.

$$
C_{B}=\frac{48 E I}{l^{3}}
$$




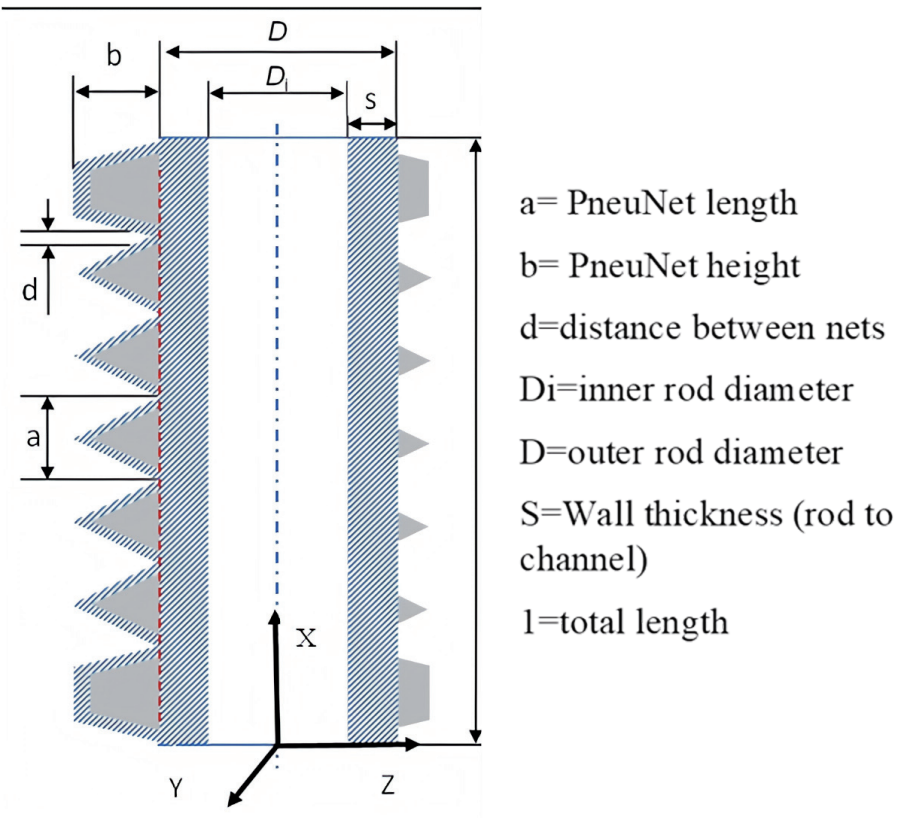

Fig. 5. Mechanical representation of the soft robotic component, explaining coordinates and labels.

under consideration of the inertia I

$$
I_{y}=I_{z}=\frac{\pi}{64}\left(D^{4}-d^{4}\right)
$$

As a first approach, the elastic modulus E can be expressed as tensile strength over fracture elongation, knowing, that the used material would reveal significant non-linearity in its elastic characteristic (visco- or hyperelastic). For PneuNets, Polygerinos et al. ${ }^{3}$ have suggested the following expression to calculate force response $\Delta \mathrm{F}$ resulting from inner pneumatic excitation $\Delta \mathrm{Pi}$

$$
\Delta F L_{W}=M_{a} \Delta P_{i}
$$

with the width- (c) justified lever $\mathrm{LW}=1 / \mathrm{c}$. The variable Ma (bending moment) takes the PneuNets pressurized dimensions into account and allows to carefully study the sensitivity towards changes in design.

$$
M_{a}=b^{2}\left(2-\frac{b^{2}}{b^{2}-(2 a-d)^{2}}\right)
$$

Because of the triangular chamber shape of the presented PneuNets, as seen in figure 5, height $b$ is justified by two thirds and $d$ is calculated as average gap over height.

\section{Experimental setup}

The behavior of the soft robotic actuator is studied under its inflation by air pressure. For the evaluation of force, it initially rests hanging from a flange mount with its tip, opposite to the excited PneuNet pointing against a force sensor. A DELTA F/T transducer force-momentum sensor, with a resolution of CTL $=1 / 8$ and NET F/T $=1 / 16$ on Fz is employed to evaluate the force, that is generated by the actuator. 


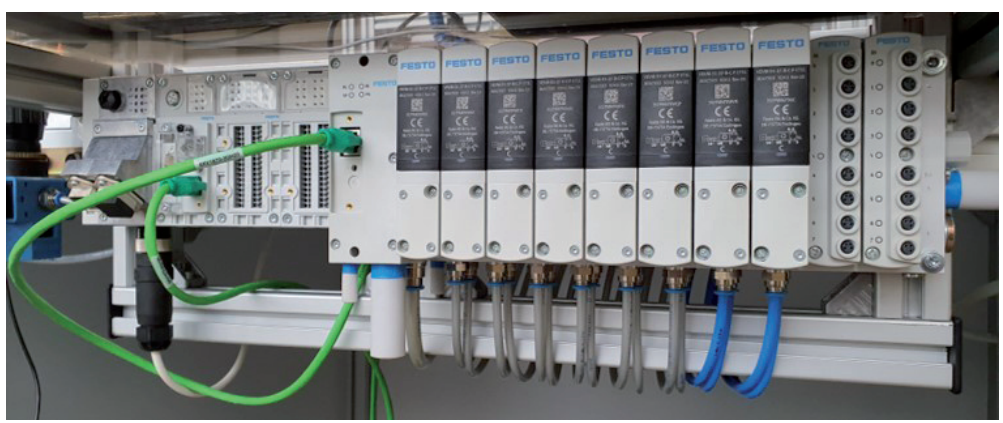

Fig. 6. FESTO Motion Terminal, with an 8-valve configuration for up to 16 parallel pneumatic outputs.

In order to supply and control pressure that for soft actuators a FESTO Motion Terminal (VTEM), ${ }^{27}$ is used (see figure 6). Implementation of a fully automated PLC environment allowed us to concentrate in our own control system signal development without the problematic that piezo actuated valves and magnetic flow valves present.

Our experimental setup can be seen in figure 7. The force sensor attached to a UR5 lightweight robot helps to easily modify the contact distance of the unpressurized and pressurized states of the actuator as in. ${ }^{24}$ For this work, only displacement and motion in one plane where captured, in future works, a threedimensional motion trajectory capture is planned.

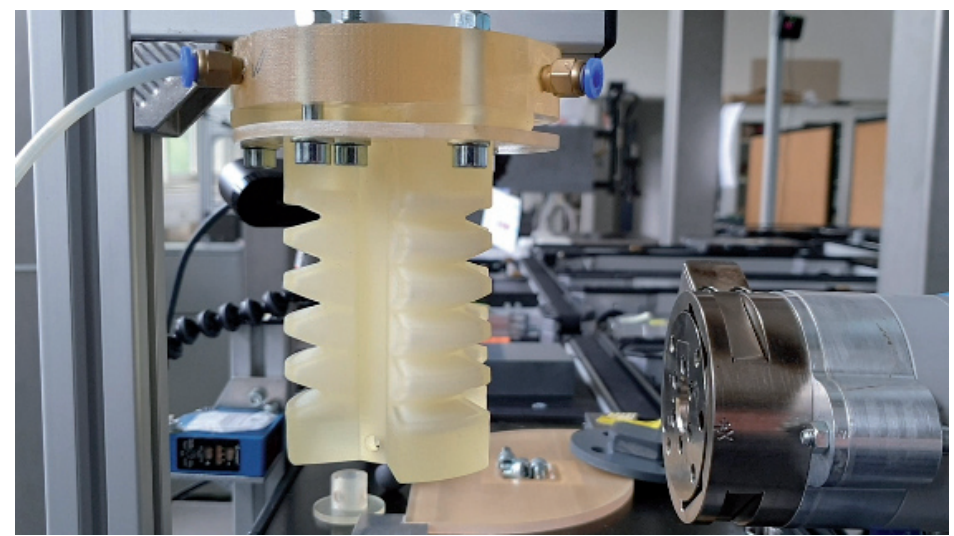

Fig. 7. Front view of the experimental setup. The actuator rest in an unpressurized state position.

The importance of this experimental setup is, to acquire enough information about if an additively manufactured actuator can provide a load force enough to be implemented in a multi-body soft robot configuration. Most commonly, soft actuators are characterized by terms of their deformation against pressurization, but as presented by, ${ }^{19}$ only a few newtons of force are obtained $(1-4 \mathrm{~N})$. For this work, a minimum expected load of $10 \mathrm{~N}$ is expected in order to carry on further experiments on an increased payload.

\section{Results and discussions}

The physical experiments of pressurizing the soft robotic actuator with a ramp signal, deliver its static response. 


\section{Bending force and momentum}

The force signal of the actuator was evaluated with a step width of 100 mbar. In figure $8 \mathrm{a}$, a force over pressure plot is presented with inflation until rupture during the last experiment.

The actuators force over pressure response shows a linear increasing trend up to 1 bar internal pressure, as predicted by the model developed in eqns. 3 and 4 . Deviations from the prediction are suspected to result the fact that the piezo valves used for this work does not react up to a minimum of $100 \mathrm{mbar}$ pressure, that explain the offset in the origin of the diagram. It was also found, that the PneuNets mechanical model shows high sensitivity against dimensioning the height parameter b. The "model 1" represent the ideal bending force mathematically calculated in comparison with the model from. ${ }^{19}$ Two tests were performed to corroborate the measured force. Results show in "Test 1 " and "Test 2 ", see figure 8 , how force values remain almost identical trough the test. The "Model" line represents an adjusted linear model of the expected ideal. In our case, during the first 130 mbar of pressurization in which the pressure controller does not react yet and due to the distance of $1 \mathrm{~mm}$ in between the force sensor attached to the robot, force cannot be measured in the interval from 0 to $130 \mathrm{mbar}$. Pressure levels going over 1 bar were starting to produce some damage on the outside walls of the actuator, meaning, that internal cracks were already propagated trough the walls of the chamber. At the point of 1.1 bar, fissures caused by extreme elongation on the zones where the networks are connected showed up as seen in figure $8 \mathrm{~b}$.

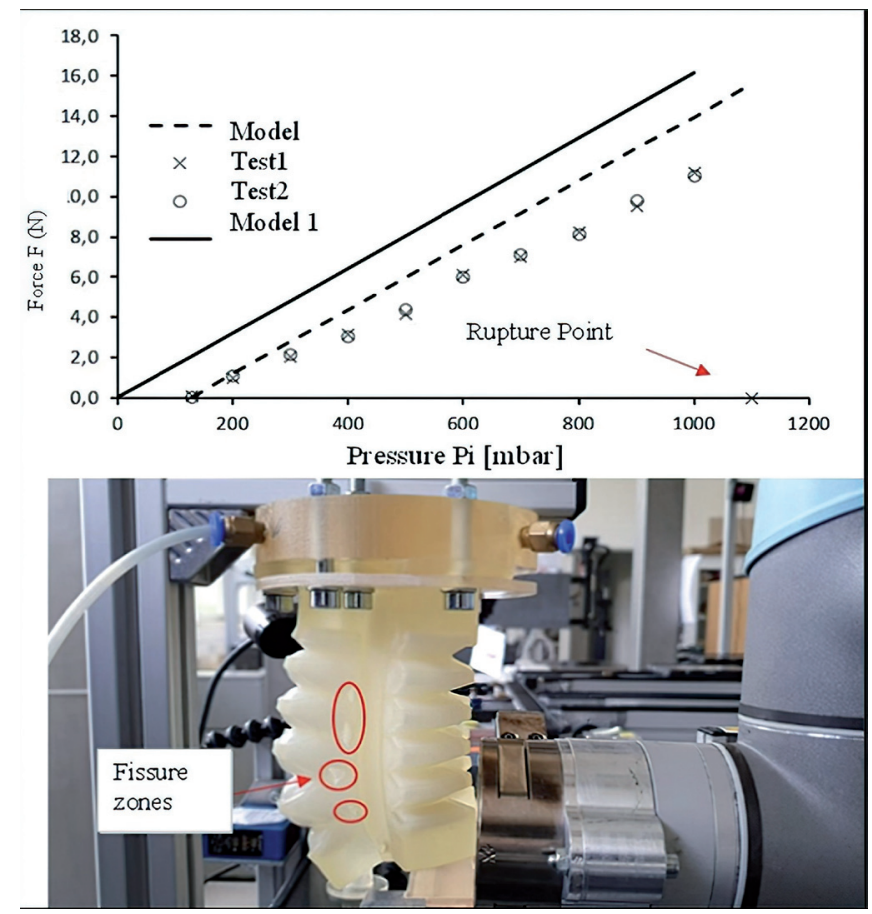

Fig. 8. a) Comparison to model at 1100 mbar pressurized state (rupture point), b) optical evaluation of the actuator begore rupture at a force state of 1100 mbar. 
Because the presented new triangular actuator design strongly diverges from the plain rod design presented in, ${ }^{19,28}$ it is still unclear which dimensioning of height to use for optimized results. The angled chambers need to be paired respect to, because this PneuNet design reasonably will generate most force at the base of the network. The height factorized by $2 / 3$ is thus chosen for a) its geometrical explanation with respect to the triangular PneuNet shape and b) the observed match of model's incline to the plots of the experiments. It can be noticed, that a quasi-linear relationship occurs in between pressure and force, with nearly $1 \mathrm{~N}$ increment each 100 mbar (see figure 9). Despite this, a supply pressure over 1 bar caused damages up to rupture on the actuator, this said, there still no chance to provide a reliable safety factor. Design parameters such as already mentioned height need to be optimized for a better expected reliabiltiy.

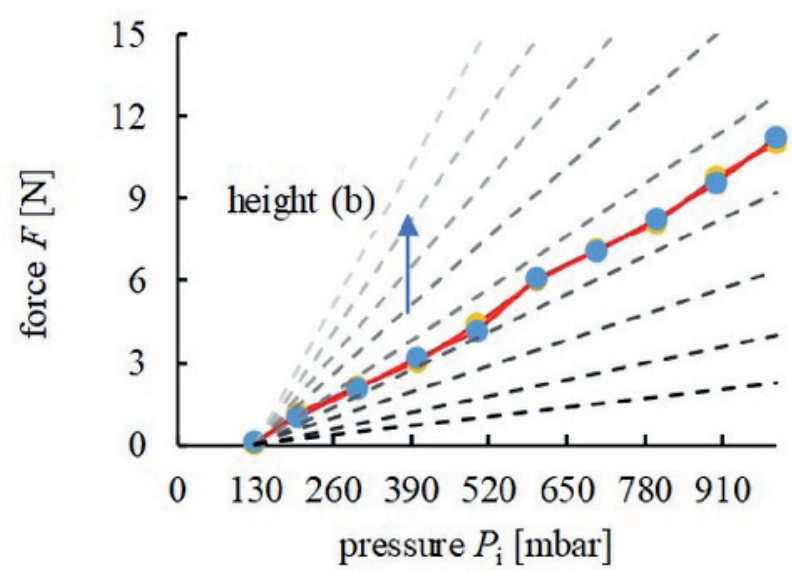

Fig. 9. $1 \mathrm{~mm}$ stepwise variation of computed chamber height illustrates the sensitivity of the mechanic model for statically pressurized PneuNets.

\section{Motion response}

Control of soft robots and actuators is mostly difficult due lack of good models that describe or represent their behavior. The complexity of such systems, has been hardly studied in recent works, such as in ${ }^{29}$ and. ${ }^{30}$ For this case, FESTO piezo pilot-controlled valves ${ }^{27}$ combined with model based proportional pressure regulation functions (i.e. ramps) are coupled with our soft actuator. The system is capable to supply up to 16 parallel air pressure continuous and cyclic signals (see figure 6) with a range of discrepancy in between the 25 mbar on each output. The linearity error for our system varies in between the 60-100 mbar and a maximum hysteresis of 25 mbar.

In contrast, for small air volumes, the PID controller has a response sensitivity of 80 mbar. Therefore, the controller is activated not before a setpoint of 100 mbar, as it can be seen in figure 10, otherwise, the port is exhausted.

Pressure sensors are directly integrated on the valves, with the model-based proportional pressure regulation, any pressure drops caused by a change in the pressure in the tubing or chambers, is calculated and compensated. Due to lack of material models and well-known problems on controlling such complex systems, this work is focused on the pure pressure regulation and control. It can be seen in figure 10 that a pressure stabilization time varies within the set point value. 


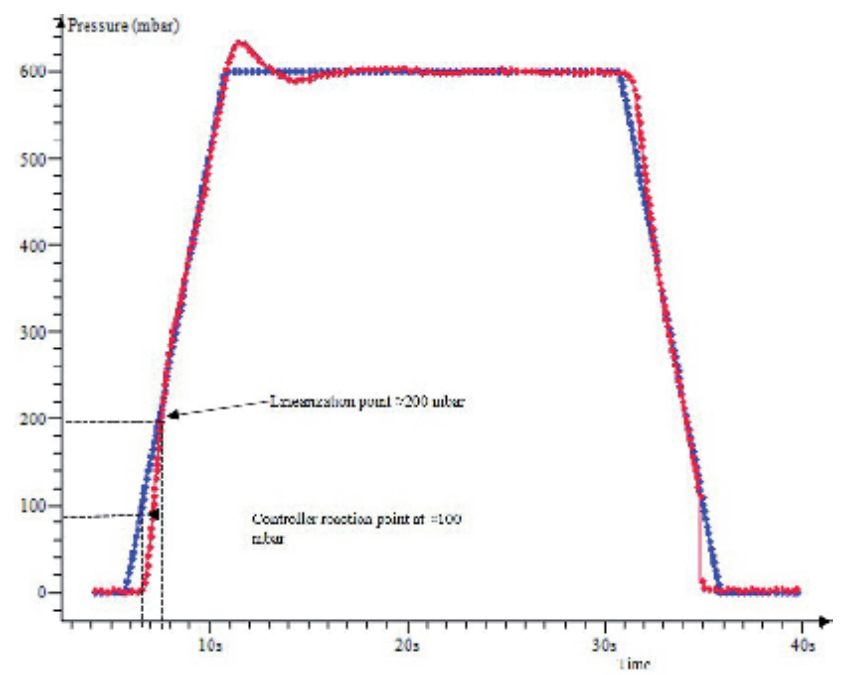

Fig. 10. ramp signal up to 600 mbar. The signal was given with a $5 \mathrm{~s}$ increment time, $20 \mathrm{~s}$ pressurized state and $5 \mathrm{~s}$ depressurization time.

For higher pressure target points, the controller tends to regulate the pressure faster than for small volumes ( $<130 \mathrm{mbar})$. In figure 10, for a target point of $600 \mathrm{mbar}$ it took approximately $8 \mathrm{~s}$ for the controller to equilibrate the signal. In contrast to our comparison model taken from, ${ }^{19}$ and due the limitations of piezo valves, the possibility to control such small air volumes is difficult.

\section{Two-Element motion test}

One of the challenges of multi-body soft manipulators is to couple each element. We design a quick-change coupling system to increase the assembly and testing agility of our manipulator system. This work presents only a bending motion overview of two independent actuators interconnected to create a two-element fully additively manufactured soft robot manipulator. As presented in figure 11, it can be seen how the bending behaviour of one independent element is affected when adding more elements to the manipulator.

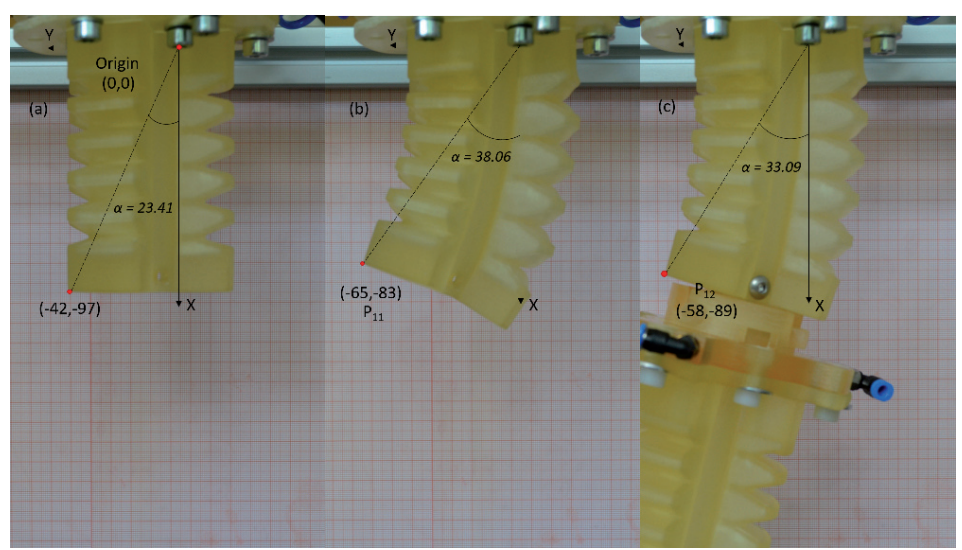

Fig. 11. Deformation coordinates in $\mathrm{mm}$ for one plane at a $400 \mathrm{mbar}$ pressure input. (a) one actuator, no pressure input; (b) one actuator, pressurized state; (c) two actuator and quick exchange coupling system, pressurized state. 
This change in motion, will seriously affect the soft robotic manipulator dynamic characteristics. Such motion influences could translate into higher air pressurization levels in a multi-body configuration to achieve similar bending angles of independent actuators. Such input parameters need to be considered during the design phase in order to avoid a component failure due to inflation levels. In future works, these changes will be further investigated.

\section{Conclusions and future work}

In this work we have presented a design approach for AM that show how a typical stepwise casting process for silicon materials can be avoided. Which lead to an easy-to manufacture product. The additive manufactured actuator was designed in consideration to being additively manufactured and actuated with a set of three internal PneuNet type chambers and channels. The results showed that is possible to get a significant amount of tip force $(11 \mathrm{~N})$ in a quasicontrolled environment. Considering that the actuator force can be increased while pressurizing more than one chamber, as not performed in this work yet, we will analyze how the simultaneous multi-axis behaves in the space and how the use of sequential signal inputs can achieve predefined primitive motions in order to implement basic pick \& place operations in zones difficult to reach (i.e., automotive cockpit cabling).

It was also demonstrated that to use this actuator altogether as a fully AM manipulator, the load force needs to be still incremented. A set of design parameters as the PneuNet height and length will be further investigated. Also, the use of a more robust actuator as the first element of our manipulator can help to increase the overall stiffness and increase maximum payload. The actuator has a mass of only $190 \mathrm{gr}$, while in a full multi-body configuration is planned to weight around $800 \mathrm{gr}$, while typically robotic arms e.g., a Universal Robot UR3 has a mass of $11.2 \mathrm{~kg}$. This can imply that the harm that classical robots represent for workers in production environments can considerably be reduced using soft materials. Furthermore, more complex control methods will be investigated for an independent signal response of each chamber through the whole manipulator and a soft AM gripper. Dynamic behaviour for a multi-element soft manipulator needs to be further investigated and evaluated in a 3D spatial configuration.

\section{REFERENCES}

1. K. Suzumori, "Development of Flexible Microactuator and Its Applications to Robotic M.".

2. C. Schumacher, B. Bickel, J. Rys, S. Marschner, C. Daraio, and M. Gross, "Microstructures to control elasticity in 3D printing," ACM Trans. Graph., vol. 34, no. 4, pp. 136:1-136:13, 2015, doi: 10.1145/2766926.

3. B. T. Phillips et al., "A Dexterous, Glove-Based Teleoperable Low-Power Soft Robotic Arm for Delicate Deep-Sea Biological Exploration," Sci. Rep., vol. 8, no. 1, pp. 1-9, 2018, doi: 10.1038/s41598-018-33138-y.

4. I.DeFalco, M. Cianchetti, and A. Menciassi, "Stiff-flop surgical manipulator: design and preliminary motion evalutaion," 4th Jt. Work. New Technol. Comput. Assist. Surg., no. October 2014, pp. 131-134, 2014, doi: 10.13140/RG.2.1.3972.9124. 
An agile multi-body additively manufactured soft actuator for soft manipulators / Jorge Morales, et al.

5. M. Hamad, N. Mansfeld, S. Abdolshah, and S. Haddadin, "The Role of Robot Payload in the Safety Map Framework," IEEE Int. Conf. Intell. Robot. Syst., pp. 195-200, 2019, doi: 10.1109/IROS40897.2019.8968022.

6. O. D. Yirmibeşoğlu, T. Oshiro, G. Olson, C. Palmer, and Y. Mengüç, "Evaluation of 3D printed soft robots in radiation environments and comparison with molded counterparts," Front. Robot. AI, vol. 6, no. MAY, pp. 1-14, 2019, doi: 10.3389/frobt.2019.00040.

7. O. D. Yirmibesoglu et al., "Direct 3D printing of silicone elastomer soft robots and their performance comparison with molded counterparts," 2018 IEEE Int. Conf. Soft Robot. RoboSoft 2018, pp. 295-302, 2018, doi: 10.1109/ ROBOSOFT.2018.8404935.

8. I. De Falco, G. Gerboni, M. Cianchetti, and A. Menciassi, "Design and Fabrication of an Elastomeric Unit for Soft Modular Robots in Minimally Invasive Surgery,” J. Vis. Exp., no. 105, pp. 1-9, 2015, doi: 10.3791/53118.

9. T. Ranzani, M. Cianchetti, G. Gerboni, I. De Falco, and A. Menciassi, "A Soft Modular Manipulator for Minimally Invasive Surgery: Design and Characterization of a Single Module," IEEE Trans. Robot., vol. 32, no. 1, pp. 187-200, 2016, doi: 10.1109/TRO.2015.2507160.

10.K. M. de Payrebrune and O. M. O'Reilly, "On constitutive relations for a rodbased model of a pneu-net bending actuator," Extrem. Mech. Lett., vol. 8, pp. 38-46, Sep. 2016, doi: 10.1016/J.EML.2016.02.007.

11.Z. Wang, R. Kanegae, S. Hirai, and W. E. T. Al, "Circular Shell Gripper for Handling Food Products," vol. 00, no. 00, pp. 1-13, 2020, doi: 10.1089/ soro.2019.0140.

12.L. Marechal, P. Balland, L. Lindenroth, F. Petrou, C. Kontovounisios, and F. Bello, "Toward a Common Framework and Database of Materials for Soft Robotics," Soft Robot., vol. 00, no. 00, 2020, doi: 10.1089/soro.2019.0115.

13. M. Manns, J. Morales, and P. Frohn, "Additive manufacturing of silicon based PneuNets as soft robotic actuators," 2018, doi: 10.1016/j.procir.2018.03.186.

14.F. Schreiber, M. Manns, and J. Morales, "Design of an additively manufactured soft ring-gripper," Procedia Manuf., vol. 28, pp. 142-147, Jan. 2019, doi: 10.1016/J.PROMFG.2018.12.023.

15. S. Rosset, O. A. Araromi, S. Schlatter, and H. R. Shea, "Fabrication Process of Silicone-based Dielectric Elastomer Actuators," J. Vis. Exp., no. 108, pp. 1-13, 2016, doi: 10.3791/53423.

16.P. Abbasi, M. A. Nekoui, M. Zareinejad, P. Abbasi, and Z. Azhang, "Position and Force Control of a Soft Pneumatic Actuator," Soft Robot., vol. 00, no. 00, pp. 1-14, 2020, doi: 10.1089/soro.2019.0065.

17.D. Rus and M. T. Tolley, "Design, fabrication and control of soft robots," Nature, vol. 521, no. 7553, pp. 467-475, 2015, doi: 10.1038/nature14543.

18. A. Raatz, S. Blankemeyer, G. Runge, C. Bruns, and G. Borchert, "Opportunities and Challenges for the Design of Inherently Safe Robots," in Soft Robotics: Transferring Theory to Application, A. Verl, A. Albu-Schäffer, O. Brock, and A. Raatz, Eds. Berlin, Heidelberg: Springer Berlin Heidelberg, 2015, pp. 173-183. 
19.P. Polygerinos et al., "Towards a soft pneumatic glove for hand rehabilitation," IEEE Int. Conf. Intell. Robot. Syst., pp. 1512-1517, 2013, doi: 10.1109/ IROS.2013.6696549.

20.B. Mosadegh et al., "Pneumatic networks for soft robotics that actuate rapidly," Adv. Funct. Mater., vol. 24, no. 15, pp. 2163-2170, 2014, doi: 10.1002/adfm.201303288.

21.J. A. Lenssen, H. Naghibi, and M. Abayazid, "Evaluation of design aspects of modular pneumatic soft robotic endoscopes," RoboSoft 2019 - 2019 IEEE Int. Conf. Soft Robot., pp. 56-61, 2019, doi: 10.1109/ ROBOSOFT.2019.8722749.

22.J. Z. Gul et al., "3D printing for soft robotics-a review," Sci. Technol. Adv. Mater., vol. 19, no. 1, pp. 243-262, 2018, doi: 10.1080/14686996.2018.143 1862.

23.M. Pérez, D. Carou, E. M. Rubio, and R. Teti, "Current advances in additive manufacturing," Procedia CIRP, vol. 88, pp. 439-444, 2020, doi: 10.1016/ j.procir.2020.05.076.

24.T. B. Tuli and M. Manns, "Hierarchical motion control for real time simulation of industrial robots," in CIRP Manufacturing Systems Conference 2019, 2019, vol. 00 .

25.K. W. A.B. Spierings, M. Voegtlin, T. Bauer, "Materials Testing Standards for Additive Manufacturing of Polymer Materials :," Prog Addit Manuf, vol. 1, pp. 9-20, 2015, doi: http://dx.doi.org/10.6028/NIST.IR.8059.

26.H.-T. Lin et al., "Softworms: the design and control of non- pneumatic, 3Dprinted, deformable robots Recent citations Softworms: the design and control of non-pneumatic, 3D-printed, deformable robots," Bioinspir. Biomim, vol. 11, 2016, doi: 10.1088/1748-3190/11/2/025001.

27.D. M. App, "Motion Terminal VTEM,” pp. 1-36, 2019.

28.K. M. de Payrebrune and O. M. O'Reilly, "On constitutive relations for a rod-based model of a pneu-net bending actuator," Extrem. Mech. Lett., vol. 8, no. 10, pp. 38-46, 2016, doi: 10.1016/j.eml.2016.02.007.

29. S. Ibrahim, J. C. Krause, and A. Raatz, "Linear and nonlinear low level control of a soft pneumatic actuator," RoboSoft 2019 - 2019 IEEE Int. Conf. Soft Robot., vol. V, pp. 434-440, 2019, doi: 10.1109/ROBOSOFT.2019.8722737.

30.Z. Gong et al., "An opposite-bending-and-extension soft robotic manipulator for delicate grasping in shallow water," Front. Robot. AI, vol. 6, no. APR, pp. 1-10, 2019, doi: 10.3389/frobt.2019.00026. 